\title{
Blue light versus green light fundus autofluorescence in normal subjects and in patients with retinochoroidopathy secondary to retinal and uveitic diseases
}

Millena Gomes Bittencourt ${ }^{1 \dagger}$, Muhammad Hassan ${ }^{2 \dagger}$, Muhammad Sohail Halim², Rubbia Afridi ${ }^{2}$, Nam V. Nguyen ${ }^{1,2}$, Carlos Plaza², Anh N. T. Tran², Mohamed Ibrahim Ahmed', Quan Dong Nguyen² and Yasir Jamal Sepah² ${ }^{2^{*}}$

\begin{abstract}
Purpose: The aim of this study is to evaluate the differences in the fundus autofluorescence (FAF) signal between the blue light autofluorescence (BAF) from Spectralis ${ }^{\circledR}$ (Heidelberg, CA) and green light autofluorescence (GAF) 200TXTM (OPTOS, UK, in normal subjects and in patients with retinochoroidopathies (RC).

Methods: In this prospective study, FAF was performed using $B L(\lambda=488 \mathrm{~nm})$ and $G L(\lambda=532 \mathrm{~nm})$ on normal subjects and patients with RC. The corresponding pairs of BAF and GAF images from both groups were analyzed using Photoshop. The strength of the FAF signal was measured on a gray scale, where optic disc was a standard to indicate absence of AF. In addition, gray values obtained from three identical points (foveal center, and points of hypo and hyper autofluorescence) in the corresponding BAF and GAF images of normal and RC subjects were divided by the optic disc value to calculate autofluorescence signal ratio $(R)$. The $R$ values at fovea $(R 1)$, hypoautofluorescent point (R2), and hyperautofluorescent point (R3) were compared between BAF and GAF modalities, in normal and in RC subjects separately.

Results: One hundred six pairs (106 eyes) of FAF images analyzed (37 pairs: normal and 69 pairs: RC subjects). In normal subjects, the mean $R 1, R 2$, and $R 3$ values for BAF were $(1.5 \pm 0.88,1.23 \pm 0.58$, and $4.73 \pm 2.85$, respectively) and for GAF were $(0.78 \pm 0.20,0.78 \pm 0.20$, and $1.62 \pm 0.39$, respectively). Similarly, in subjects with $R C$, the mean $R 1, R 2$, and $R 3$ values for BAF were $(1.68 \pm 1.02,1.66 \pm 1.15$, and $7.75 \pm 6.82$, respectively) and for GAF were $(0.95 \pm 0.59,0.79 \pm 0.45$, and $2.50 \pm 1.65$, respectively). The mean difference in the $R 1, R 2$, and $R 3$ ratios between BAF and GAF in normal and in RC subjects was statistically significant $(p<0.001)$. The strength of the correlation $(r)$ between ratios for BAF and GAF was weak or not statistically significant in both normal and RC subjects $(p>0.05)$.

Conclusion: The distribution and intensity of the AF signal differ in BAF and GAF and cannot be used interchangeably. In BAF, optic disc signal is always weaker than in other areas, which was not true for GAF where optic disc signal was stronger than fovea and hypoautofluorescent point in both groups.
\end{abstract}

Keywords: Retinal imaging, Fundus autofluorescence, Autofluorescence imaging, Blue-light autofluorescence, Greenlight autofluorescence

\footnotetext{
* Correspondence: yjs@stanford.edu

Millena Gomes Bittencourt and Muhammad Hassan share the first authorship.

${ }^{\dagger}$ Millena Gomes Bittencourt and Muhammad Hassan contributed equally to this work.

${ }^{2}$ Byers Eye Institute, Stanford University, 2370 Watson Court, Suite 200, Palo

Alto, CA 94303, USA

Full list of author information is available at the end of the article
} 


\section{Introduction}

In recent years, the study of retinal fundus autofluorescence (FAF) has provided important information regarding the production of retinal fluorophores during physiological aging and in pathological events [1]. Through noninvasive examination techniques, FAF images can map the metabolic status of both retinal pigment epithelium (RPE) and photoreceptor outer segment.

It is well known that the dominant source of FAF signal results from light excitation of the fluorophores in lipofuscin (LF) $[2,3]$. LF is physiologically produced within the RPE and reflects its metabolic activity, which is largely determined by the quantity of photoreceptor outer segment renewal [4]. Among the many bisretinoid LF fluorophores, vitamin A-derived pyridinium-bis-retinoid (A2-E) is one of the most studied components. A2E along with other fluorophores are subject to photooxidation and photodegradation with resulting damage to RPE secondary to formation of advanced glycosylated end products, complement activation, detergent like effect on lysosomal membranes, impaired lysosomal activity, and generation of free radicals within the RPE cells [5-8].

The fluorescence emission from these fluorophores has a broad range which peaks at approximately $\lambda 610 \mathrm{~nm}[2$, 9]. However, these bisretinoids have different excitation maxima's in the visible spectrum including $\lambda 430 \mathrm{~nm}$ (alltrans-retinal dimer), $\lambda 439 \mathrm{~nm}$ (A2E), $\lambda 426 \mathrm{~nm}$ (iso-A2E), $\lambda 490 \mathrm{~nm}$ (A2-dihydropyridine-phosphatifylethanolamine, A2-DHP-PE), and $\lambda 510 \mathrm{~nm}$ (all-trans-retinal dimer-phosphatidylethanolamine and all-trans-retinal dimer-ethanolamine) [10-13]. It was also noted that as the excitation wavelength increases, the spectral width of emission spectrum decreases [3].

Such wide range of excitation spectra means that the fluorophores responsible for autofluorescence (AF) in the FAF imaging can vary depending on which excitation wavelength is used. The main excitation light more commonly used in commercial devices has been the blue light (BL) ( $\lambda 488 \mathrm{~nm})$. However, more recently, green light (GL) ( $\lambda 514 \mathrm{~nm}$ and $\lambda 532 \mathrm{~nm}$ ) were introduced for clinical use in commercial confocal scanning laser ophthalmoscopes (cSLO) and adapted ultra-wide-field retinal imaging systems. In addition to potentially exciting different fluorophores, GL is less absorbed by macular pigments compared to $\mathrm{BL}$ and enhances LF signal in macula [14].

Despite the proximity of BL and GL within the light spectrum, a potential difference in FAF signal is yet to be determined in images originated by various commercial devices. Thus, a detailed quantification of the blue light AF (BAF) and green light AF (GAF) signal will facilitate precise interpretation of AF signals in a clinical context. The index study is a prospective investigation with the purpose of evaluating the differences in the
FAF signal obtained by the BL used in Spectralis ${ }^{\circ}$ HRA + OCT (Heidelberg Engineering Inc., Vista, CA, USA) and GL used in P200Tx (OPTOS Inc., Dunfermline, Scotland, UK), and to provide a better understanding of the relationships between the measurements obtained from each device.

\section{Methods}

The index study enrolled normal subjects and patients with an established diagnosis of retinochoroidopathy (RC) secondary to uveitis and other retinal diseases who were being followed at a tertiary care ophthalmology clinic. The study was conducted in compliance with the declaration of Helsinki, US Code of Federal Regulations Title-21, and the Harmonized Tripartite Guidelines for Good Clinical Practice (1996). The study was approved by the local Institutional Review Board. A written informed consent was obtained from all participants. All normal subjects underwent fundus examination to document the health of their retina. Any subject with media opacity was excluded from the study.

\section{Inclusion and exclusion criteria}

Patients were included in the study if they met the following criteria: (1) availability of BAF and GAF images of gradable quality, (2) no lesion involving the optic disc which can affect the optic disc fluorescence, and (3) patients with retinochoroidopathy should have lesions within the posterior pole.

\section{Fundus autofluorescence imaging}

After pupillary dilation with topical tropicamide and phenylephrine, BAF images were taken using the Spectralis ${ }^{\circ}$ HRA + OCT (Heidelberg Engineering Inc., Vista, CA, USA) after subjects were kept in dark room for $30 \mathrm{~min}$. No other imaging was performed on the study subjects prior to fundus autofluorescence (FAF). Spectralis uses an optically pumped solid-state blue laser $(\lambda 488 \mathrm{~nm})$ for excitation and uses a barrier filter to capture fundus emissions above $\lambda 500 \mathrm{~nm}$ of the spectrum. FAF images of a rectangular $30^{\circ} \times 30^{\circ}$ field of view were recorded with an ametropic corrector. To improve the signal-to-noise ratio, nine images were aligned and an average image with $768 \times 768$ pixels was calculated with the Spectralis ${ }^{\circ}$ HRA + OCT software (Heidelberg Eye Explorer, v4.3; Heidelberg Engineering, Germany). After a gap of $30 \mathrm{~min}$ to account for photobleaching, green light FAF (GAF) images were taken using the Optomap-af function in $100^{\circ}$ RexMax mode of P200Tx (OPTOS Inc., Dunfermline, Scotland, UK). A single GAF image was acquired using green light $(\lambda 532 \mathrm{~nm})$ for excitation and by capturing fundus emissions between $\lambda 570$ and $780 \mathrm{~nm}$ of the spectrum. The central 
$30^{\circ} \times 30^{\circ}$ field was manually obtained using the $\mathrm{V} 2^{\circ}$ Vantage software.

\section{FAF image registration and autofluorescence signal ratio} The pairs of BAF and GAF were co-registered and analyzed using Photoshop (V S5, Adobe Systems Inc., San Jose, CA, US). Only the central $30^{\circ} \times 30^{\circ}$ images were used for analysis. The BL and GL images were re-sized to the same pixel/inch rate and aligned based on various retinal landmarks such as retinal vessels. The strength of the FAF signal was defined by the absolute intensity in a gray scale which ranges from 1 to 256 . The value 1 is considered the absolute black color possible and 256 the absolute white color possible. By definition, low pixel values (dark) represent low intensities of autofluorescence signal, and high pixel values (bright) represent high intensities of AF signal.

The optic disc AF intensity was used as a standard to indicate the absence of fluorescence, in both BAF and GAF. Multiple corresponding points (3-5) were selected in the optic disc area of both BAF and GAF images and their intensities were averaged. In addition, the optic nerve head served as an index of background noise. Three points of interest were identified in both BAF and GAF images of normal and RC subjects. These points included the foveal center, one point in an area of hypoautofluorescence, and one in an area of hyperautofluorescence. The software ruler and retinal landmarks were used to guarantee the measurement of the same points in both BAF and GAF images.

To compare the AF signal strength between the optic disc and the points of interest, the gray values measured in the three identified points (Foveal center, hypoautofluorescent, and hyperautofluorescent points) were divided from the values in the optic disc (Fig. 1), to compute the AF signal ratio $(R)$ in both BAF and GAF images of the normal and RC subjects. The $R$ values were calculated and used in this study to account for difference in the two imaging devices in terms of confocality, image capture, and image processing methods. The $R$ values were labeled as $R 1, R 2$, and $R 3$ for foveal center, hypoautofluorescent, and hyperautofluorescent points, respectively.

In normal subjects, the hypoautofluorescent point selected was in the vessels and the hyperautofluorescent point was chosen in the area 70-150 $\mu \mathrm{m}$ from the fovea. Similarly, in the subjects with RC, a point was selected in the hypoautofluorescent lesion and another point was selected in the hyperautofluorescent lesion.

\section{Blue light FAF vs green light FAF}

The $R 1, R 2$, and $R 3$ values were compared to test the agreement and the strength of correlation between BAF and GAF modalities, in both the normal and RC subjects.

\section{Statistical analysis}

The SPSS (IBM ${ }^{\circ}$ Inc., Chicago, IL) release 19.0.0 was used for statistical analysis. Demographic characteristics of the patients were summarized using descriptive statistics and expressed as mean and standard deviations. The mean $R$ values were calculated for points of interest in both BAF and FAF eyes. To test the agreement between $R$ values of BAF and GAF at same location, the mean difference and standard deviation were calculated, and Bland-Altman scatter plots were generated. To verify the strength of the correlation, the Pearson coefficients were used.

\section{Results}

A total of 106 pairs (106 eyes) of FAF images were included in this prospective study. Each pair consisted of BAF and GAF images of the same area. Thirty-seven pairs (37 eyes) of the images were from the normal subjects. Seventy-eight pairs (78 eyes) were obtained from

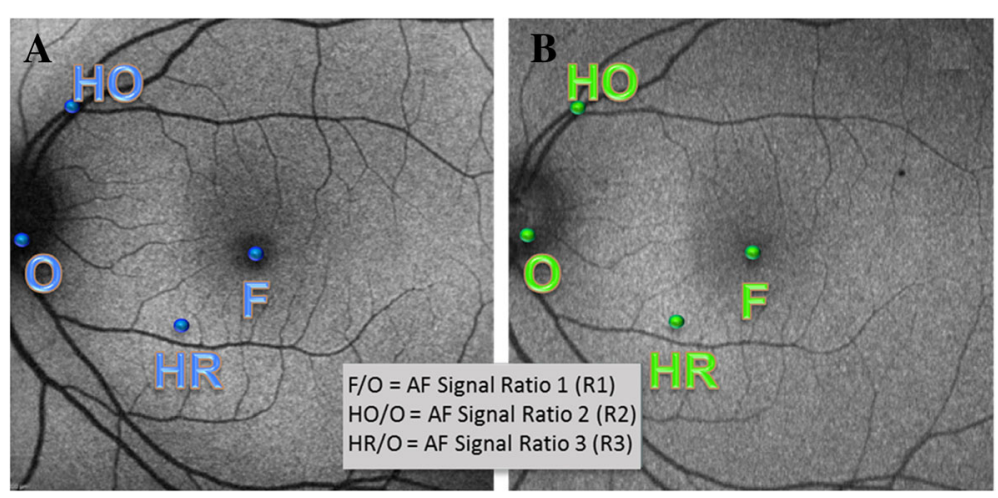

Fig. 1 Blue light and green light fundus autofluorescence images with points of interest identified. Fundus autofluorescence images acquired with blue light (a) and green light (b) in a normal subject showing the identical points of interest measured in both images. For both images, the gray values obtained in the fovea (F), hypoautofluorescent point $(\mathrm{HO})$, and hyperautofluorescent point (HR) were divided from the gray values in the optic disc $(O)$ to calculate the autofluorescence signal ratios $R 1, R 2$, and $R 3$ respectively 
subjects with various RCs. Table 1 outlines the demographic characteristics of the study population.

\section{Autofluorescence signal ratio for BAF and GAF in normal subjects}

The average $R$ in the fovea (R1) of normal subjects was $1.5 \pm 0.88$ and $0.78 \pm 0.20$ as determined by BAF and GAF, respectively (Fig. 2). Similarly, the average $R 2$ in the vessels was $1.23 \pm 0.58$ and $0.61 \pm 0.20$ for BAF and GAF images, respectively. Finally, the average $R 3$ in the point located $70-150 \mu \mathrm{m}$ from the fovea was $4.73 \pm 2.85$ and $1.62 \pm 0.39$ as assessed by BAF and GAF, respectively (Table 2). Figure 2 plots the $95 \%$ confidence interval distribution across for ratios measured by BAF and GAF in the normal subjects.

\section{Autofluorescence signal ratio for BAF and GAF in RC subjects}

In the group of eyes with RCs, the average $R$ values followed the same trend seen in normal subjects. The average $R 1$ in $R C$ subjects was $1.68 \pm 1.02$ and $0.95 \pm$ 0.59 as determined by BAF and GAF in the GL FAF images. Similarly, the average $R 2$ in point within hypofluorescent lesion was $1.66 \pm 1.15$ and $0.79 \pm 0.45$ for BAF and GAF images, respectively. Finally, the average $R 3$ in the point located within hyperfluorescent lesion was $7.75 \pm 6.82$ and $2.50 \pm 1.65$ as assessed by BAF and GAF, respectively (Table 2). Figure 2 plots the 95\% confidence interval distribution across for ratios measured by BAF and GAF in the RC subjects.

\section{Agreement between BAF and GAF autofluorescence signal ratios}

The mean difference in the $R 1, R 2$, and $R 3$ ratios between BAF and GAF in normal eyes was statistically significant ( $R 1: 0.72 \pm 0.9, p<0.0001$ (Fig. 3)); $R 2: 0.62 \pm$ 0.52, $p<0.0001 ; R 3: 3.10 \pm 2.81, p<0.0001$ ) (Table 3). Similar results were also observed in eyes with RCs, where difference in the $R 1, R 2$, and $R 3$ ratios between BAF and GAF was $0.73 \pm 1.09(p<0.0001), 0.86 \pm 1.05$ $(p<0.0001)$, and $4.76 \pm 6.56(p<0.0001)$, respectively (Table 3). The bland-Altman scatterplots for differences between BAF and GAF ratios in normal and RC subjects are shown in Fig. 4. The scatterplots show no agreement between the AF signal ratios of BAF and GAF images, with a statistically significant difference between both groups.

Table 1 Demographic characteristics of the study population

\begin{tabular}{|c|c|c|c|c|c|}
\hline Diagnosis & Number of eyes & Mean age years (SD) & Gender (F:M) & Ethnicity & Number of pair of images \\
\hline \multicolumn{6}{|l|}{ Normal subjects with no known ocular disease } \\
\hline Normal subjects & 37 (17 subjects) & $32( \pm 7)$ & $17 \mathrm{~F}: 14 \mathrm{M}$ & 9C: $2 \mathrm{AA}$ & 37 \\
\hline \multicolumn{6}{|l|}{ Retinochoroidopathy secondary to retinal diseases } \\
\hline High myopia & 13 & $29( \pm 6.43)$ & $11 \mathrm{M}: 2 \mathrm{~F}$ & $9 A: 4 C$ & 13 \\
\hline Hydroxychloroquine retinal toxicity & 2 & $38(N / A)$ & $2 \mathrm{~F}$ & $2 C$ & 2 \\
\hline Aged-related macular degeneration (drusen) & 1 & $74(N A)$ & $1 \mathrm{M}$ & $1 \mathrm{C}$ & 1 \\
\hline Central serous chorioretinopathy & 2 & $48( \pm 8.66)$ & $2 \mathrm{M}$ & $2 \mathrm{~A}$ & 2 \\
\hline Cone-rod dystrophy & 4 & $75( \pm 0.57)$ & $4 \mathrm{M}$ & $2 \mathrm{C}: 2 \mathrm{~A}$ & 4 \\
\hline Diabetic retinopathy & 2 & $68(N / A)$ & $2 \mathrm{M}$ & $2 C$ & 2 \\
\hline Sickle cell retinopathy & 1 & $48(N / A)$ & $1 \mathrm{~F}$ & $1 \mathrm{AA}$ & 1 \\
\hline Lymphoma (intraocular) & 1 & $74(\mathrm{~N} / \mathrm{A})$ & $1 \mathrm{M}$ & $1 C$ & 1 \\
\hline \multicolumn{6}{|l|}{ Uveitic retinochoroidopathies } \\
\hline Panuveitis (idiopathic) & 1 & $30(N / A)$ & $1 \mathrm{~F}$ & $1 \mathrm{C}$ & 1 \\
\hline Vogt-Koyanagi Harada & 2 & $25(N / A)$ & $2 \mathrm{M}$ & $2 \mathrm{AA}$ & 2 \\
\hline Acute zonal occult outer retinopathy & 3 & $44( \pm 21)$ & $3 F$ & $3 C$ & 3 \\
\hline Punctate inner choroidopathy & 10 & $31( \pm 6.67)$ & $8 F: 2 M$ & $10 C$ & 10 \\
\hline Birdshot choroidoretinopathy & 5 & $54( \pm 4.54)$ & $3 F: 2 M$ & $5 C$ & 5 \\
\hline Multifocal choroiditis & 16 & $40( \pm 8.03)$ & $16 \mathrm{~F}$ & 12C: 2AA: 2A & 16 \\
\hline Sarcoidosis with posterior uveitis & 2 & $83(N / A)$ & $2 \mathrm{~F}$ & $2 C$ & 2 \\
\hline Serpiginous choroiditis & 2 & $38(N / A)$ & $2 \mathrm{M}$ & $2 \mathrm{C}$ & 2 \\
\hline Retinochoroiditis of unclear etiology & 2 & $31(N / A)$ & $2 \mathrm{~F}$ & $2 \mathrm{AA}$ & 2 \\
\hline Total & 69 (42 patients) & $41( \pm 16)$ & $38 \mathrm{~F}: 31 \mathrm{M}$ & 47C: 15A: 7AA & 69 \\
\hline
\end{tabular}

Age: $S D$ standard deviation and $N / A$ not applicable; gender: $F$ female and $M$ male; ethnicity: $C$ Caucasian, $A A$ African-American, and $A$ Asian 

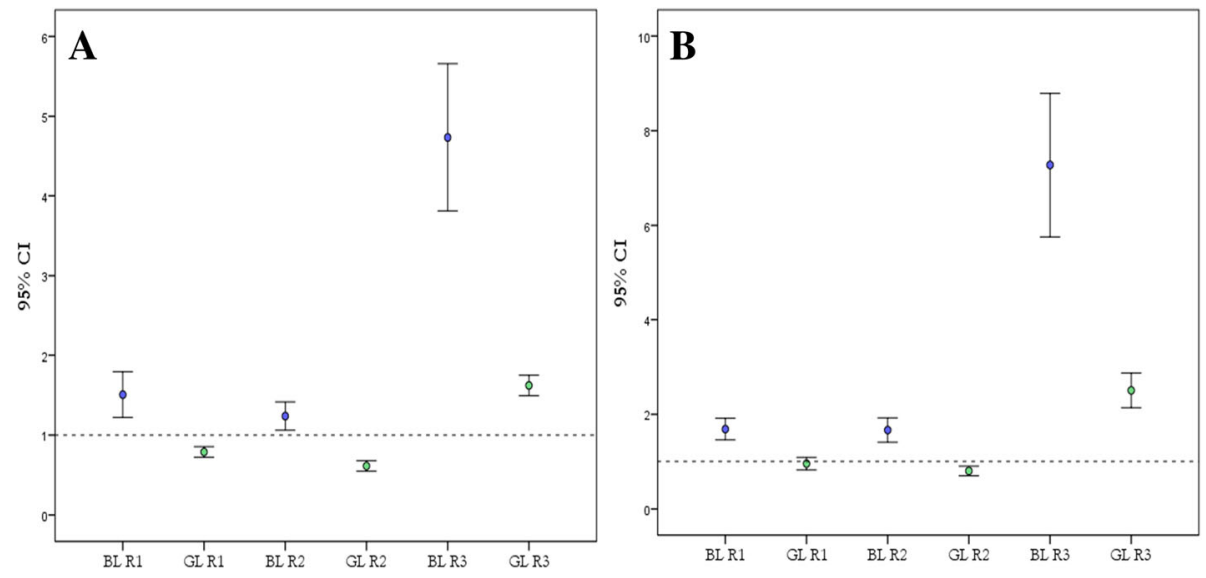

Fig. 2 Confidence interval plots. Confidence interval plots for autofluorescence signal ratios $(R)$ measured in normal (a) and RC (b) subjects. The $R$ values are represented by blue and green circle for BAF and GAF, respectively. The $R$ values above dotted lines represent signals more intense than the optic disc signal (dotted line). Similarly, below the dotted are located the AF signals weaker than the signal in the optic disc

\section{Correlation between BAF and FAF autofluorescence signal ratios}

The strength of the correlation $(r)$ between $R$ values for BAF and GAF was weak or not statistically significant in both normal eyes $(R 1 r=0.015, p>0.05 ; R 2 r=0.308$, $p>0.05$; and $R 3 r=0.158, p>0.05)$ and $R C$ eyes $(R 1 r=$ $0.179, p>0.05$; $R 2 r=0.407, p>0.05$; and $R 3 r=0.276$, $p>0.05)$.

\section{Discussion}

With the advent of cSLO, FAF images have left the research laboratories and have become more popular as a propaedeutic tool to assist in the diagnosis, management, and monitoring of certain conditions. However, despite its incontestable importance, FAF image interpretation can be particularly challenging and are subject to individual interpretation of gray intensity and contrast between normal an abnormal area. Many papers have

Table 2 Autofluorescence signal ratio $(R)$ in normal and RC subjects

\begin{tabular}{|c|c|c|c|c|c|c|}
\hline \multicolumn{7}{|c|}{ Autofluorescence signal ratio $(R)$} \\
\hline & \multicolumn{3}{|c|}{ Blue light $(\lambda 488 \mathrm{~nm})$} & \multicolumn{3}{|c|}{ Green light $(\lambda 532 \mathrm{~nm})$} \\
\hline & Mean & SD & $\begin{array}{l}95 \% \text { Confidence } \\
\text { interval }\end{array}$ & Mean & SD & $\begin{array}{l}\text { 95\% Confidence } \\
\text { interval }\end{array}$ \\
\hline \multicolumn{7}{|c|}{ Normal subjects } \\
\hline$R 1$ & 1.50 & 0.88 & $1.26-1.83$ & 0.78 & 0.20 & $0.72-0.85$ \\
\hline$R 2$ & 1.23 & 0.54 & $0.36-2.61$ & 0.61 & 0.20 & $0.54-0.67$ \\
\hline R3 & 4.73 & 2.85 & $3.93-5.76$ & 1.62 & 0.39 & $1.50-1.74$ \\
\hline \multicolumn{7}{|c|}{ RC subjects } \\
\hline$R 1$ & 1.68 & 1.02 & $1.48-1.91$ & 0.95 & 0.59 & $0.83-1.09$ \\
\hline$R 2$ & 1.66 & 1.15 & $1.42-1.93$ & 0.79 & 0.45 & $0.70-0.89$ \\
\hline R3 & 7.27 & 6.82 & $5.98-8.78$ & 2.50 & 1.65 & $2.19-2.86$ \\
\hline
\end{tabular}

$S D$ standard deviation qualitatively characterized the FAF patterns and their progression in various retinal pathologies.

Lois and colleagues were among the first to quantitatively assess the differential distribution of FAF signal in the images obtained with cSLO in normal volunteers and in patients [15]. Their work was primarily based on BL FAF images and did not include other wavelengths. As a first effort to explore the difference between blue and green excitatory lights, Wolf-Schnurrbusch et al. evaluated differences in size of geographic atrophy lesion as assessed by BL $(\lambda 488 \mathrm{~nm})$ and GL $(\lambda 514 \mathrm{~nm})$ using a modified cSLO (HRAmp; Heidelberg Engineering) [16]. There was a significant difference in measurements of GA lesion size between the two wavelengths. Additionally, GAF was better at delineation of borders of atrophic patches in foveal region and preserved foveal island. Similarly, another study demonstrated slightly larger GA lesion size of GAF compared to BAF with significantly higher intergrader reliability in GAF compared to BAF [17]. Both of these findings were attributed to better contrast offered by the GAF. These findings show that wavelength used to acquire the images may play a role in the visualization of lesions.

Our group proposed a method similar to the one described by Lois et al. to assess the strength of AF signals and to evaluate the relation, in this case ratio, between BL and GL FAF [15]. However, unlike the method of Lois et al., which subtracts the optic nerve head intensity from the retinal signal, our method estimates the ratio between AF signals in various retinal areas and optic disc and does not entail the primary purpose of describing the distribution of the AF in degrees of eccentricity. Additionally, using the $R$ values allows us to compare two different imaging devices and account for their differences in terms of confocality, image capture, and 

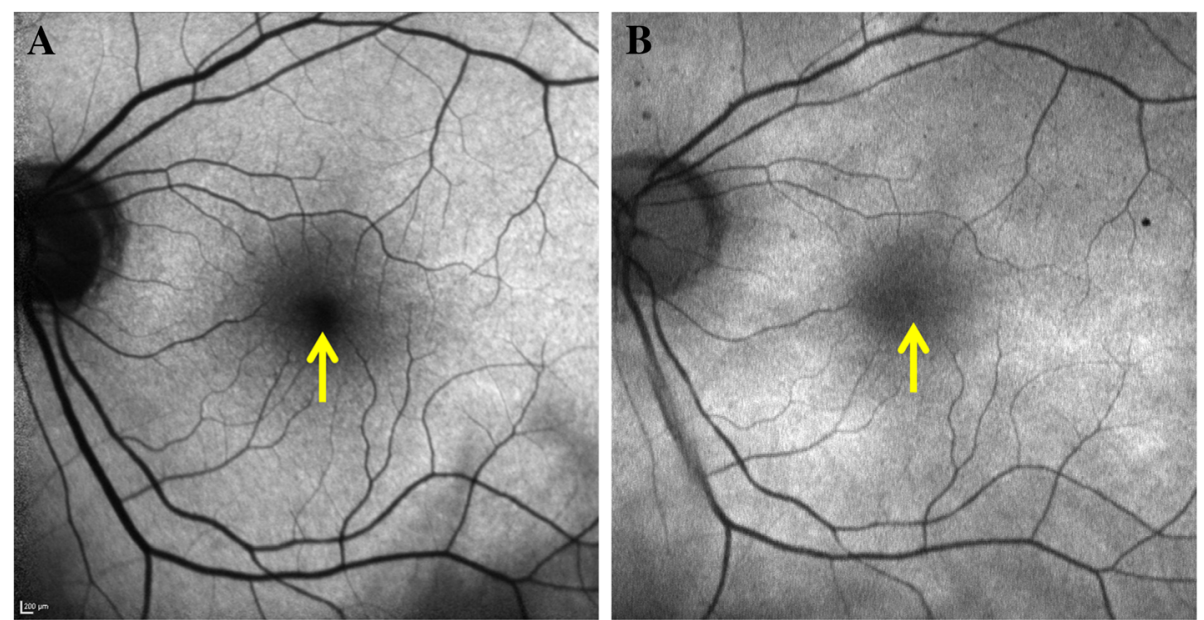

Fig. 3 Blue light vs green light autofluorescence signals at the fovea. Blue light autofluorescence (BAF) (a) and green light autofluorescence (GAF) (b) images at the foveal center demonstrating the differences in the foveal autofluorescence (AF) signal. The AF signal due to BAF (a) appears much darker compared to the GAF (b) as the blue light is more strongly absorbed by the macular pigments compared to the green light

image processing methods. In our study, the relation between optic disc and fovea $(R 1)$ hypo $(R 2)$ and hyperautofluorescent $(R 3)$ areas was consistent across normal subjects and subjects with RC within same modality. However, the $R$ values were not equivalent across BAF and GAF images. The agreement and the correlation of both FAF modalities were significantly different supporting the findings seen by Wolf-Schnurrbusch and Pfau et al. $[16,17]$.

In BAF imaging of the normal eyes, the gray intensity in the optic nerve head consisted of dark black due to the absence of LF in this area. Similarly, the blood vessels showed a weak strength of the AF signal due to absorption of BL by hemoglobin, yet it was higher than the optic disc. Normally in the BAF, the foveal center appears hypoautofluorescent due to absorption of $\mathrm{BL}$ by luteal pigment and melanin. However, the AF signal as detected by our study was

Table 3 Difference between autofluorescence signal ratios $(R)$ measured by blue light and green light at the fovea (R1), hypoautofluorescent point (R2), and hyperautofluorescent point $(R 3)$

\begin{tabular}{lccc}
\hline & Mean difference & SD & Sig. $(p)$ \\
\hline $\begin{array}{l}\text { Difference between blue and green ratios: normal subjects } \\
\text { images) }\end{array}$ N $=39$ \\
R1 Difference & 0.72 & 0.90 & 0.000 \\
$R 2$ Difference & 0.62 & 0.52 & 0.000 \\
$R 3$ Difference & 3.10 & 2.81 & 0.000 \\
Difference between blue and green ratios: patients ( $N=80$ images) \\
R1 Difference & 0.73 & 1.09 & 0.000 \\
$R 2$ Difference & 0.86 & 1.05 & 0.000 \\
$R 3$ Difference & 4.76 & 6.56 & 0.000 \\
\hline SD standard deviation & & &
\end{tabular}

$S D$ standard deviation still brighter than the optic disc. In GAF imaging of normal eyes, the AF signal at the optic disc was higher than at the foveal center even though normally the AF signal at fovea is higher in GAF due to weaker absorption of GL by macular pigment compared to the foveal AF signal in BAF as BL is stronger absorbed by the macular pigments (Fig. 3) [18]. Similarly, the AF signal at optic disc in GAF was higher than that of the blood vessels. In RC eyes, the $R$ values for BAF and GAF signal followed similar pattern to normal eyes. Figure 5 describes a case demonstrating difference in the AF signal of a hypoautofluorescent lesion as captured by BAF and GAF. The optic disc had the lowest AF signal in BAF images compared to fovea and even hypoautofluorescent lesions. On the other hand, optic disc AF signal intensity was higher in GAF images compared to fovea and hypoautofluorescent lesions. The difference in the autofluorescent pattern of the optic disc between BAF and GAF can be because of excitation of different fluorophores by the two wavelengths (Fig. 6). These differences in the AF signals captured by BAF and GAF underscore the importance of not using the FAF images captured by the Heidelberg Spectralis and Optos P200Tx interchangeably.

In our study, we also noted that the range of the confidence interval of the $R$ values also differed between BAF and GAF modalities. The intervals were broader in the BAF images compared to the GAF images. Sparrow et al. demonstrated that emission spectra of the fluorophores present in the LF varied depending on the wavelength used [3]. As the wavelength increased, the emission spectral width decreased considerably from $190 \mathrm{~nm}$ at $\lambda 330 \mathrm{~nm}$ to $60 \mathrm{~nm}$ at $\lambda 545 \mathrm{~nm}$. Therefore, 

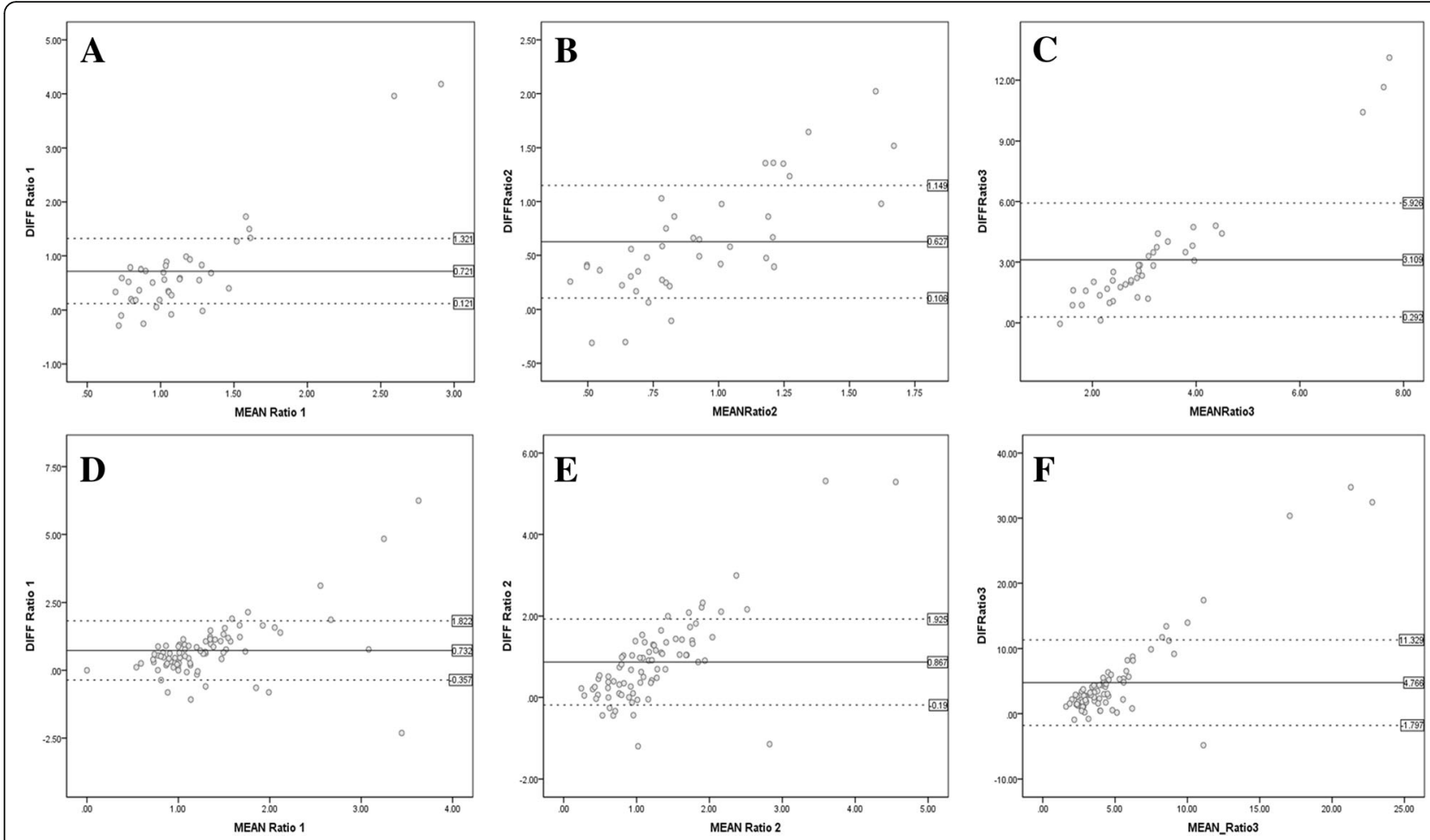

Fig. 4 Bland-Altman scatterplots. Bland-Altman scatterplots for differences between BAF and GAF autofluorescence ratios (R) in normal (a fovea; $\mathbf{b}$ hypoautofluorescent point; $\mathbf{c}$ hyperautofluorescent point) and RC (d fovea; e hypoautofluorescent point; $\mathbf{f}$ hyperautofluorescent point) subjects

the variance in emission intensities may be responsible to a wider confidence interval of gray scale measurements in BAF noted in our study.

Our study has provided valuable insight about FAF image analysis. A variety of diagnoses, from retinal vascular to uveitic diseases, although small in number, provide a diverse sampling of how BAF and GAF may differ among different entities. However, our study also contained some limitations. The absolute values of the AF signal could not be used for comparison due to the
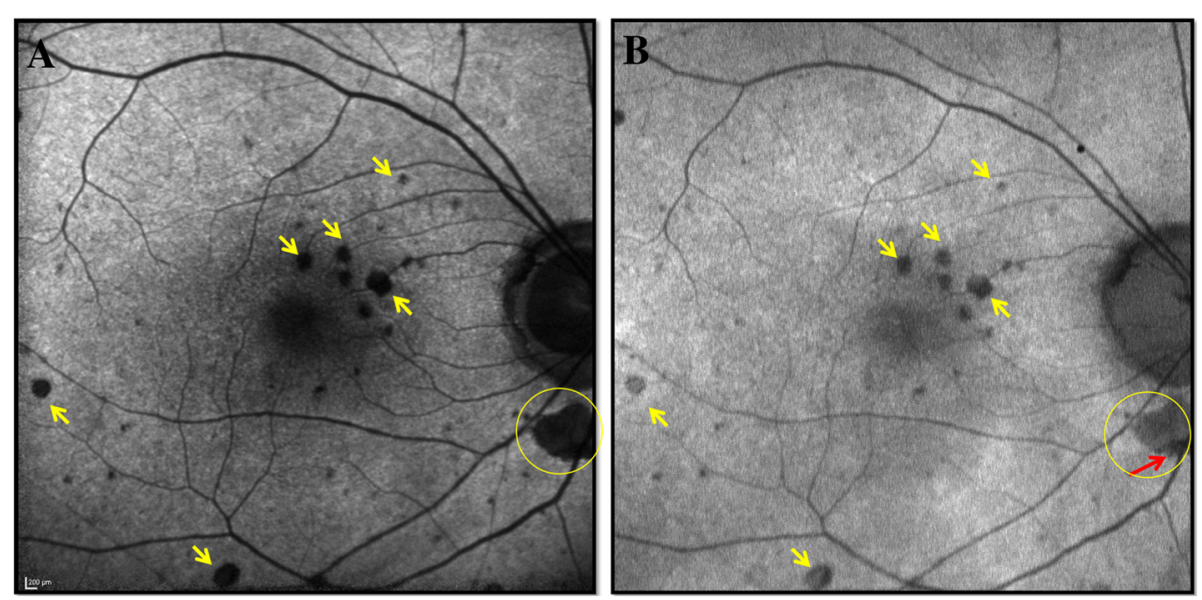

Fig. 5 A case outlining discrepancy in blue and green light autofluorescence signals of similar hypoautofluorescent lesions in posterior uveitis. Blue light autofluorescence (BAF) (a) and green light autofluorescence (GAF) (b) images of an 83-year-old Caucasian woman with posterior uveitis secondary to sarcoidosis (retinochoroiditis), showing hypoautofluorescent lesions (yellow arrows) spread throughout the posterior pole. The fundus autofluorescence images show noticeable discrepancies in the autofluorescence AF signals of the hypofluorescent lesions captured by the BAF (a) and GAF (b). Additionally, in the lesion inferior to the optic nerve (yellow circle, red arrow), there is a small area of deep loss of AF signal within the hypoautofluorescent lesion as shown by the GAF (b). The detail is not revealed by the BAF image 

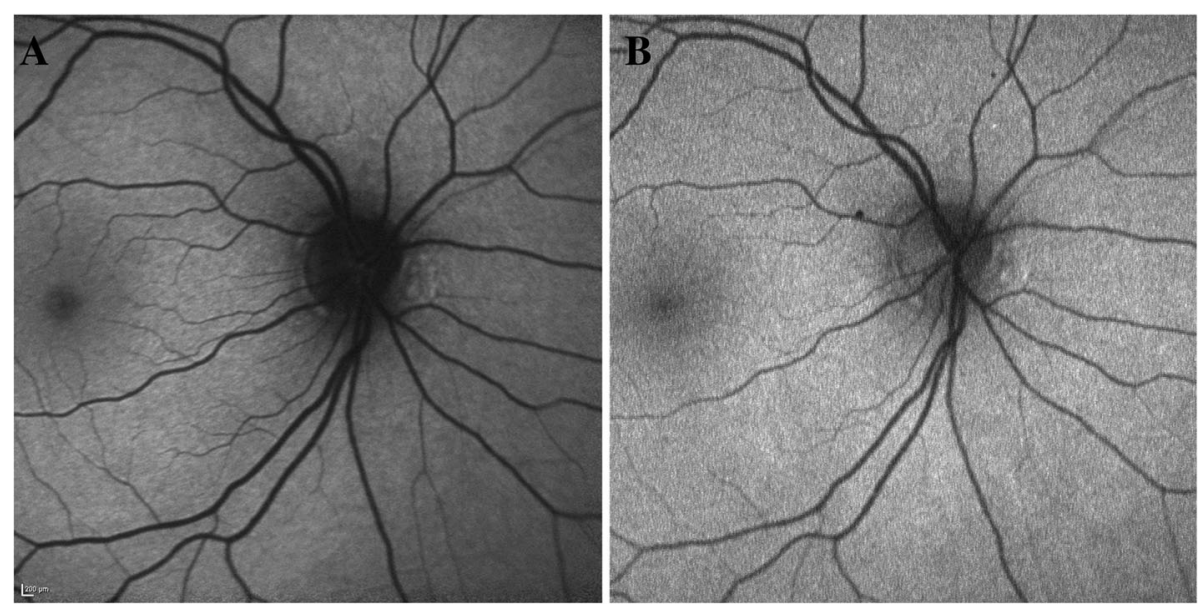

Fig. 6 Blue light vs green light autofluorescence signals at the optic disc. Blue light autofluorescence (BAF) (a) and green light autofluorescence (GAF) (b) images of optic disc. There are noticeable discrepancies in the autofluorescence signal at the optic disc as captured by BAF (a) and GAF (b)

difference in the optics of both devices, translating in different intensity of fundus exposure, gain, and distortions. Also, the sample size of our study was relatively small, especially in specific categories.

\section{Conclusion}

The quantitative evaluation of AF signal is an important step toward the correct interpretation of BAF and GAF images in clinical practice. According to our results, the distribution and intensity of the FAF signal differ in BAF and GAF images acquired by Heidelberg Spectralis ${ }^{\circ}$ and Optos P200Tx ${ }^{\mathrm{Tn}}$, respectively. Therefore, the outcomes from these two devices are not simply interchangeable despite using wavelengths which are in close proximity in visual spectrum. The difference was consistent across normal and RC subjects. Further analyses are indicated to confirm if the images from Spectralis ${ }^{\circ}$ and P200Tx ${ }^{\mathrm{Tm}}$ may provide similar or different AF characterization of diseases. Moreover, the discrepancies in our findings underscore the importance of evaluating the consequences of design choices made by the manufacturers.

\section{Abbreviations}

AF: Autofluorescence; BAF: Blue-light autofluorescence; BL: Blue light; FAF: Fundus autofluorescence; GAF: Green-light autofluorescence; GL: Green light; LF: Lipofuscin; R: Autofluorescence signal ratio;

RC: Retinochoroidopathy; RPE: Retinal pigment epithelium

\section{Acknowledgements}

Not applicable.

\section{Funding}

There was no funding received for this study.

\section{Availability of data and materials}

The datasets used during the current study are available from the corresponding author on reasonable request.

\section{Authors' contributions}

$\mathrm{MB}$ contributed in the study concept and design, acquisition of data, manuscript drafting, and revising. $\mathrm{MH}$ contributed in the study concept and design, data analysis and interpretation, manuscript drafting, review, and revising. MSH contributed in the analysis and interpretation, manuscript review, and revision. RA contributed in the manuscript drafting. NN contributed in the manuscript review and revision. CP contributed in the manuscript review and revision. AT contributed in the manuscript drafting and revision. MIA contributed in the study concept and design, data analysis. QDN contributed in the study concept and design, interpretation, manuscript revision, and approval to publish. YJS contributed in the study concept and design, manuscript revision, and final approval to publish. All authors read and approved the final manuscript.

\section{Ethics approval and consent to participate}

The study was conducted in compliance with the declaration of Helsinki, US Code of Federal Regulations Title-21, and the Harmonized Tripartite Guidelines for Good Clinical Practice (1996). The study was approved by the local Institutional Review Board. A written informed consent was obtained from all participants.

\section{Consent for publication}

Not applicable.

\section{Competing interests}

YJS has received research support from Astellas, Genentech, and Optovue, and serves on the Scientific Advisory Board for Genentech/Roche, Optos, and Regeneron.

QDN is a recipient of a Physician Scientist Award from Research to Prevent Blindness, New York, NY, and serves on the Scientific Advisory Board for AbbVie, Bayer, Genentech, Regeneron, and Santen, among others. QDN also chaired the Steering Committee for the RISE and RIDE studies and was on the Steering Committee for the VISTA Study, and other studies sponsored by Genentech and Regeneron.

No other authors have received any financial funding or support.

\section{Publisher's Note}

Springer Nature remains neutral with regard to jurisdictional claims in published maps and institutional affiliations.

\section{Author details}

${ }^{1}$ Ocular Imaging Research and Reading Center, Menlo Park, CA, USA. ${ }^{2}$ Byers Eye Institute, Stanford University, 2370 Watson Court, Suite 200, Palo Alto, CA 94303, USA. 
Received: 23 September 2018 Accepted: 20 December 2018

Published online: 08 January 2019

References

1. Bindewald A, Bird AC, Dandekar SS, Dolar-Szczasny J, Dreyhaupt J, Fitzke FW, Einbock W, Holz FG, Jorzik JJ, Keilhauer C et al (2005) Classification of fundus autofluorescence patterns in early age-related macular disease. Invest Ophthalmol Vis Sci 46:3309-3314.

2. Delori FC, Dorey CK, Staurenghi G, Arend O, Goger DG, Weiter JJ (1995) In vivo fluorescence of the ocular fundus exhibits retinal pigment epithelium lipofuscin characteristics. Invest Ophthalmol Vis Sci 36:718-729.

3. Sparrow JR, Wu Y, Nagasaki T, Yoon KD, Yamamoto K, Zhou J (2010) Fundus autofluorescence and the bisretinoids of retina. Photochem Photobiol Sci 9: 1480-1489.

4. Sparrow JR, Boulton M (2005) RPE lipofuscin and its role in retinal pathobiology. Exp Eye Res 80:595-606.

5. Vives-Bauza C, Anand M, Shiraz AK, Magrane J, Gao J, Vollmer-Snarr HR, Manfredi G, Finnemann SC (2008) The age lipid A2E and mitochondrial dysfunction synergistically impair phagocytosis by retinal pigment epithelial cells. J Biol Chem 283:24770-24780.

6. Ben-Shabat S, Itagaki Y, Jockusch S, Sparrow JR, Turro NJ, Nakanishi K (2002) Formation of a nonaoxirane from $\mathrm{A} 2 \mathrm{E}$, a lipofuscin fluorophore related to macular degeneration, and evidence of singlet oxygen involvement. Angew Chem Int Ed Engl 41:814-817.

7. Yoon KD, Yamamoto K, Ueda K, Zhou J, Sparrow JR (2012) A novel source of methylglyoxal and glyoxal in retina: implications for age-related macular degeneration. PLoS One 7:e41309.

8. Zhou J, Jang YP, Kim SR, Sparrow JR (2006) Complement activation by photooxidation products of A2E, a lipofuscin constituent of the retina pigment epithelium. Proc Natl Acad Sci U S A 103:16182-16187.

9. Delori FC, Goger DG, Dorey CK (2001) Age-related accumulation and spatial distribution of lipofuscin in RPE of normal subjects. Invest Ophthalmol Vis Sci 42:1855-1866.

10. Parish CA, Hashimoto M, Nakanishi K, Dillon J, Sparrow J (1998) Isolation and one-step preparation of $\mathrm{A} 2 \mathrm{E}$ and iso-A2E, fluorophores from human retinal pigment epithelium. Proc Natl Acad Sci U S A 95:14609-14613.

11. Fishkin NE, Sparrow JR, Allikmets R, Nakanishi K (2005) Isolation and characterization of a retinal pigment epithelial cell fluorophore: an all-transretinal dimer conjugate. Proc Natl Acad Sci U S A 102:7091-7096.

12. Kim SR, Jang YP, Jockusch S, Fishkin NE, Turro NJ, Sparrow JR (2007) The alltrans-retinal dimer series of lipofuscin pigments in retinal pigment epithelial cells in a recessive Stargardt disease model. Proc Natl Acad Sci U S A 104: 19273-19278.

13. Wu Y, Fishkin NE, Pande A, Pande J, Sparrow JR (2009) Novel lipofuscin bisretinoids prominent in human retina and in a model of recessive Stargardt disease. J Biol Chem 284:20155-20166.

14. Ravera V, Giani A, Pellegrini M, Oldani M, Invernizzi A, Carini E, Cigada M, Bottoni F, Staurenghi G (2017) Comparison among different diagnostic methods in the study of type and activity of choroidal neovascular membranes in age-related macular degeneration. Retina. 2017:0:1-7.

15. Lois N, Halfyard AS, Bird AC, Fitzke FW (2000) Quantitative evaluation of fundus autofluorescence imaged "in vivo" in eyes with retinal disease. $\mathrm{Br}$ J Ophthalmol 84:741-745

16. Wolf-Schnurrbusch UE, Wittwer W, Ghanem R, Niederhaeuser M, Enzmann V, Framme C, Wolf S (2011) Blue-light versus green-light autofluorescence: lesion size of areas of geographic atrophy. Invest Ophthalmol Vis Sci 52: 9497-9502.

17. Pfau M, Goerdt L, Schmitz-Valckenberg S, Mauschitz MM, Mishra DK, Holz FG, Lindner M, Fleckenstein M (2017) Green-light autofluorescence versus combined blue-light autofluorescence and near-infrared reflectance imaging in geographic atrophy secondary to age-related macular degeneration. Invest Ophthalmol Vis Sci 58:Bio121-bio130.

18. Snodderly DM, Brown PK, Delori FC, Auran JD (1984) The macular pigment. I. Absorbance spectra, localization, and discrimination from other yellow pigments in primate retinas. Invest Ophthalmol Vis Sci 25:660-673.

\section{Submit your manuscript to a SpringerOpen ${ }^{\circ}$ journal and benefit from:}

- Convenient online submission

- Rigorous peer review

- Open access: articles freely available online

- High visibility within the field

- Retaining the copyright to your article

Submit your next manuscript at $\boldsymbol{\nabla}$ springeropen.com 